Professora Titular da Escola de Humanidades (Letras) e do Programa de Pós-Graduação em Letras do Sul.

https://orcid.org/0000-0002-9638-1180

E-mail: cperna@pucrs.br

2 Professor Associado no Departamento de Filologia Inglesa da Universidad de Sevilla, Espanha.

https://orcid org/0000-0003-2908-7261

E-mail:mpadillacruz@us.es

Doutoranda em Linguística na Pontifícia Universidade Católica do Rio Grande do Sul Brasil. https://orcid.org/0000-0002-5577-2214

E-mail: gisellerevisora@gmail.com

Recebido em: 05/11/2019.

Aprovado em: 05/11/2019.

Publicado em: 27/12/2019.

Endereço:

Cristina Lopes Perna

(6) 6681, Partenon, Porto Alegre-RS,

A0619-900

C/S. Fernando, 4, C.P. 41004, Sevilla, Espanha

Giselle Liana Fetter

Dernardes, 116, Cachoeirinha-RS 94920-150

\section{Pragmática da interlíngua e pragmática instrucional: dois campos de pesquisa vibrantes e elucidativos}

Cristina Lopes Perna ${ }^{1}$

Pontifícia Universidade Católica do Rio Grande do Sul, Escola de Humanidades, Porto Alegre, RS, Brasil.

Manuel Padilla Cruz ${ }^{2}$

Universidad de Sevilla, Filología Inglesa, Sevilla, Espanha.

Giselle Liana Fetter ${ }^{3}$

Pontificia Universidade Católica do Rio Grande do Sul, Escola de Humanidades, Porto Alegre, RS, Brasil.

Dentre as áreas oriundas da ampla disciplina da pragmática estão a pragmática da interlíngua e a pragmática instrucional Inspirada por um enorme campo de investigação transcultural realizado no final dos anos 1980 e no início dos anos 1990, e instituída por nomes conhecidos, como Gabriele Kasper, Shoshana Blum-Kulka e Juliane House, para citar apenas alguns, a pragmática da interlíngua é tradicionalmente definida como o estudo sobre a aquisição, o desenvolvimento e a operacionalização de conhecimentos de L2 de aprendizes (KASPER; ROSE, 2001). Desde a sua criação, essa vertente mais profícua da pragmática tem sido intrinsecamente vinculada à aquisição de segunda língua, logo, a pesquisa centrou-se nas habilidades comunicativas dos alunos de L2, nos problemas que eles enfrentam em determinadas circunstâncias e nas falhas e êxitos ao usar a(s) língua(s)-alvo para diversas finalidades (KASPER; BLUM-KULKA, 1993; KASPER; SCHMIDT, 1996). Fundamentados por diversas estruturas e abordagens teóricas (KASPER, 2001), e recorrendo a distintos métodos empíricos, especialistas desse campo dedicaram os seus esforços à investigação de um grande número de questões relacionadas à produção verbal com o objetivo de compreender o que os alunos realmente expressam ao realizarem certas ações com interlocutores específicos em determinados contextos (BARDOVI-HARLIG, 2013).

Se há uma área que atraiu a atenção de pragmáticos da interlíngua foi, provavelmente, a realização de ações verbais pelos alunos em situações reais ou simuladas. Consequentemente, há muitos estudos sobre os atos de fala como os cumprimentos (JAWORSKI, 1994), as desculpas (OLSHTAIN, 1983; BLUM-KULKA; LEVENTSON, 1987; PERNA, 2002), as solicitações (BLUMKULKA; LEVENTSON, 1987; BLUM-KULKA, 1988; FÆRCH; KASPER, 1989) ou a expressão de gratidão (HARLOW, 1990; EISENSTEIN; BODMAN, 1993). No entanto, muitos outros fenômenos comunicativos e idiossincrasias do comportamento comunicativo dos 
alunos em sua respectiva L2 não foram desconsiderados por especialistas nesse campo. É suficiente citar os estudos sobre a falha pragmática (THOMAS, 1983; BEEBE; TAKAHASHI, 1989), transferência pragmática (KASPER, 1992), (im) polidez e trabalho relacional (HINKEL, 1996; LOCASTRO, 1997), gerenciamento conversacional (KÖNIG, 2013; SHIVELY, 2015) ou o uso de marcadores pragmáticos ou discursivos (ROMERO TRILLO, 2002; GÁNEM-GUTIÉRREZ; ROEHR, 2011). Por outro lado, os trabalhos que abordam a compreensão de L2 são menos numerosos, embora não por esse motivo sejam menos esclarecedores ou úteis. Alguns exemplos notáveis são os estudos sobre os efeitos dos marcadores de discurso na compreensão dos alunos (CHAUDRON; RICHARDS, 1986), os problemas que eles têm na dedução de conteúdos implícitos (TAGUCHI, 2009, 2013) ou ao lidarem com expressões idiomáticas (COOPER, 2012), as suas percepções do comportamento do ato de fala (WOODFIELD, 2012) ou as violações pragmáticas (BARDOVI-HARLIG, DÖRNYEI, 2012) e as suas habilidades de vigilância epistêmica (PADILLA CRUZ, 2013; IFANTIDOU, 2014).

Mesmo que a pragmática instrucional tenha uma história relativamente recente, ela se desenvolveu como uma inevitável ramificação ou como uma consequência da pragmática da interlíngua. 0 pressuposto subjacente, e talvez motivador, à sua criação parece ter sido que, se os alunos de L2 obviamente enfrentarem dificuldades ao colocar em prática os seus conhecimentos abstratos de L2 e as suas habilidades comunicativas, o seu desempenho certamente se beneficiará da instrução (ISHIHARA; COHEN, 2010). Portanto, o vínculo entre essas duas vertentes da pragmática parece óbvio: a pragmática da interlíngua informa a pragmática instrucional sobre os problemas dos alunos ao usar e processar a L2, bem como sobre as dificuldades que algumas áreas específicas podem representar para eles, e a pragmática instrucional desenvolve e fornece ferramentas para melhorar a consciência e o desempenho metapragmático dos alunos de L2. Por conseguinte, a pragmática instrucional pode ser caracterizada como um ramo da pragmática que se ocupa da implementação de procedimentos pedagógicos destinados a empreender ou a melhorar o ensino de aspectos pragmáticos específicos da L2 (ISHIHARA, 2010; LOUW; DERWING; ABBOTT, 2010; ABRAMS, 2014). Seu escopo, no entanto, não pode simplesmente se limitar a esse empreendimento valioso, já que estudiosos da área também abordaram questões tais como o ensino da pragmática de L2, os efeitos de diversas intervenções, o ensino explícito e implícito, os benefícios e as desvantagens da dedução e indução de métodos pedagógicos, o impacto das experiências de estudo no exterior ou da formação de professores (ALCÓN SOLER, 2005; SAFONT JORDÁ, 2005; TAKAHASHI, 2005; ISHIHARA, 2010; TAGUCHI, 2015).

Esses dois campos de estudo não puderam escapar da atenção da Revista Letrônica, que dedicou esta edição especial atual a eles. Sob o tema abrangente da "Pragmática da Interlíngua e Pragmática Instrucional", a edição pretende mostrar a sua vivacidade e apresentar alguns dos recentes interesses, preocupações e trabalhos realizados em cada um deles. A edição reúne sete contribuições, de âmbitos distintos, de pesquisadores de diferentes universidades. Essas contribuições são organizadas conforme a ordem na qual foram nomeadas as duas áreas em foco no título da edição. Assim, os trabalhos são divididos em dois grupos. 0 primeiro inclui quatro trabalhos dedicados à pragmática da interlíngua, enquanto o segundo compreende os três restantes, que se enquadram no campo da pragmática instrucional.

O primeiro artigo do primeiro grupo é de Giovani Santos, e é intitulado "Second language pragmatics: a corpus-based study of the pragmatic marker like". O trabalho aborda o uso desse marcador pragmático "like" por estudantes universitários brasileiros residentes na Irlanda e compara as suas funções interpessoais e significados processuais aos do marcador equivalente usado por falantes nativos de inglês irlandês. 0 segundo artigo, "Ordens e pedidos em língua italiana: um estudo de percepção de falantes nativos e aprendizados brasileiros", é de autoria conjunta de Luciane Nascimento Spadotto e Elisabetta Santoro. Com foco nas percepções dos alunos, o artigo investiga as características de pedidos e solicitações em italiano e examina os critérios pelos quais os falantes nativos e os alunos brasileiros de italiano os diferenciam. "O inglês para aviação e pragmática: uma proposta de investigação descritiva por meio da Linguística de Corpus" é o terceiro artigo desse grupo. Fundamentado na pragmática de corpus, Malila Carvalho de Almeida Prado procura elucidar alguns elementos comunicativos importantes, empregados pelos profissionais da aviação e os motivos subjacentes às suas escolhas para conscientizar os futuros profissionais. Por fim, o quarto artigo é "Mobile instant messaging: a bonanza for interlanguage pragmatics" de contrbuição de Bethany Aull. Após delinear a atual situação da pesquisa sobre esse tipo de comunicação, a autora argumenta que pode ser interessante e útil a pesquisa em pragmática 
da interlíngua devido às possibilidades de evidenciar a interseção de culturas e comunidades mediadas eletronicamente.

Quanto ao segundo grupo, o primeiro trabalho apresenta uma proposta pedagógica. De autoria de Manuel Padilla Cruz, "Dealing with jokes in the ESL class: a pedagogical proposal centred on comprehension" propõe cinco etapas para abordar piadas em inglês na sala de aula de ESL e sensibilizar os alunos para os elementos nos quais o seu humor é contingente. 0 segundo e o terceiro artigo, por outro lado, direcionam a atenção para a avaliação do impacto da instrução pragmática. Em "Investigating the effects of pragmatic instruction: a comparison of L2 Spanish compliments and apologies during short term study-abroad", Megan DiBartolomeo, Vanessa Elias e Daniel Jung analisam o desenvolvimento desses dois atos de fala expressivos em estudantes que completaram cinco semanas do programa de estudos no exterior em Mérida, no México, mas que receberam apenas instruções sobre o primeiro desses atos de fala. Da mesma forma, Pauline Madella e Jesús Romero-Trillo examinam o efeito da instrução em "Prosodic pointing in inferential comprehension: the application of Relevance Theory to L2 listening instruction". Esse artigo mostra que, ao introduzir o que os autores chamam de "apontamento prosódico" - definido como um fenômeno ostensivo que inclui características paralinguísticas vocais e visuais e é usado de forma síncrona para comunicar intenções -, os alunos chineses de L2 podem ajudálos a ajustar a sua vigilância epistêmica em inglês como L2.

Certamente, as análises apresentadas em alguns dos artigos aqui reunidos podem elucidar outras investigações e fornecer dados valiosos para a comparação e o estudo de outras questões. Esperamos que as considerações expostas em trabalhos da área e/ou os problemas que eles salientam desencadeiem mais pesquisas em um desses dois campos vibrantes da pragmática. Também esperamos que as propostas pedagógicas oferecidas venham a inspirar outras que possam facilitar o ensino de questões pragmáticas de L2 ou que resultem em melhorias significativas dessas que já existem. Esse futuro trabalho certamente beneficiará a aquisição e o ensino de segundas línguas.

\section{Referências}

ABRAMS, Zsuzsanna I. Using film to provide a context for teaching L2 pragmatics. System, v. 46, p. 55-64, Oct. 2014. https://doi.org/10.1016/j.system.2014.06.005

ALCÓN SOLER, Eva. Does instruction work for learning pragmatics in the EFL context? System, V. 33, n 3, p. 417-435, Sep. 2005. https://doi.org/10.1016/j.system.2005.06.005

BARDOVI-HARLIG, Kathleen. Developing L2 pragmatics. Language Learning, v. 63, n 1, p. 68-86, Feb. 2013. https://doi.org/10.1111/j.1467-9922.2012.00738.x

BARDOVI-HARLIG, Kathleen; DÖRNYEI, Zoltán. Do language learners recognize pragmatic violations? Pragmatic versus grammatical awareness in instructed L2 learning. TESOL Quarterly, v. 32, n 2, p. 233-259, Jan. 2012. https://doi.org/10.2307/3587583

BEEBE, Leslie M.; TAKAHASHI, Tomoko. Do you have the bag? Social status and patterned variation in second language acquisition. In: GASS, Susan; MADDEN, Carolyn; PRESTON, Dennis; SELINKER, Larry (ed.) Variation in Second Language Acquisition (Vol. 1: Discourse and pragmatics). Clevedon: Multilingual Matters, 1989. p. 103-125. https://doi.org/10.1017/ $\underline{\text { s0272263100009591 }}$

BLUM-KULKA, Shoshana. Interpreting and performing speech acts in a second language: A cross-cultural study of Hebrew and English. In: WOLFSON, Nessa; JUDD, Elliot (ed.) Sociolinguistics and Language Acquisition. Rowley: Newbury House, 1988. p. 36-55. https:// doi.org/10.1177/007542428702000112

BLUM-KULKA, Shoshana; LEVENSTON, Edward A. Lexical-grammatical pragmatic indicators. Studies in Second Language Acquisition, v. 9, n 2, p. 155-170, Jun. 1987. https://doi.org/10.1017/ $\underline{\mathrm{s} 0272263100000450}$

CHAUDRON, Craig; RICHARDS, Jack C. The effect of discourse markers on the comprehension of lectures. Applied Linguistics, v. 7, n 2, p. 113-127, Jul. 1986. https://doi.org/10.1093/applin/7.2.113

COOPER, Thomas C. Processing of idioms by L2 learners of English. TESOL Quarterly, v. 33, n 2, p. 233-262, Jan. 2012. https://doi.org/10.2307/3587719

EISENSTEIN, Miriam; BODMAN, Jean. Expressing gratitude in American English. In: KASPER, Gabriele; BLUM-KULKA, Shoshana (ed.). Interlanguage Pragmatics. New York: Oxford University Press, 1993. p. 64-81. https://doi.org/10.1017/s0047404500018649 
Færch, Claus; KASPER, Gabriele. Internal and external modification in interlanguage request realisation. In: HOUSE, Juliane; BLUM-KULKA, Shoshana; KASPER, Gabriele (ed.) Cross-cultural Pragmatics: Requests and Apologies. Norwood: Ablex, 1989. p. 221-247. https://doi.org/10.1017/ $\underline{\mathrm{s} 0272263100010846}$

GÁNEM-GUTIÉRREZ, Gabriela A.; ROEHR, Karen. Use of L1, metalanguage, and discourse markers: L2 learners' regulation during individual task performance. International Journal of Applied Linguistics, v. 21, n 3, p. 397-318, Nov. 2011. https://doi.org/10.1111/i.1473-4192.2010.00274.x

HARLOW, Linda L. Do they mean what they say? Sociopragmatics competence and second language learners. The Modern Language Journal, v. 74, n 3, p. 328-351, 1990. https://doi. org/10.1111/j.1540-4781.1990.tb01070.x

HINKEL, Eli. When in Rome: Evaluations of L2 pragmalinguistic behaviors. Journal of Pragmatics, v. 26, n 1, p. 51-70, Jul. 1996. https://doi.org/10.1016/0378-2166(95)00043-7

IFANTIDOU, Elly. Pragmatic Competence and Relevance. Amsterdam: John Benjamins, 2014.

ISHIHARA, Noriko. Instructional pragmatics: Bridging teaching, research, and teacher education. Language and Linguistics Compass, v. 4, n. 10, p. 938-953, Oct. 2010. https://doi. org/10.1111/j.1749-818x.2010.00242.x

ISHIHARA, Noriko; COHEN, Andrew D. Teaching and Learning Pragmatics. Where Language and Culture Meet. Harlow: Pearson Education, 2010. https://doi.org/10.1111/j.1540-4781.2012.01348.x

JAWORSKI, Adam. Pragmatic failure in a second language: Greeting responses in English by Polish students. International Review of Applied Linguistics in Language Teaching, v. 32, n 1, p. 41-55, 1994. https://doi.org/10.1515/iral.1994.32.1.41

KASPER, Gabriele. Pragmatic transfer. Second Language Research, v. 8, n 3, p. 203-231, Oct. 1992.

KASPER, Gabriele. Four perspectives on L2 pragmatic development. Applied Linguistics, v. 22, n 4, p. 502-530, Dec. 2001. https://doi.org/10.1093/applin/22.4.502

KASPER, Gabriele; BLUM-KULKA, Shoshana. Interlanguage Pragmatics: An Introducction. New York: Oxford University Press, 1993. https://doi.org/10.1017/s0047404500018649

KASPER, Gabriele; ROSE, Kenneth R. (ed.). Pragmatics in Language Teaching. Cambridge: Cambridge University Press, 2001.

KASPER, Gabriele; SCHMIDT, Richard. Developmental issues in interlanguage pragmatics. Studies in Second Language Acquisition, v. 18, n 2, p. 149-169, Jun. 1996. https://doi.org/10.1017/ $\underline{\mathbf{s} 0272263100014868}$
KÖNIG, Clelia. Topic management in French L2: A longitudinal conversation analytic study EUROSLA Yearbook, v. 13, n 1, p. 226-250, Jan. 2013. https://doi.org/10.1075/eurosla.13.11kon

LoCASTRO, Virginia. Politeness and pragmatic competence in foreign language education. Language Teaching Research, v. 1, n 3, p. 239-267, Sep. 1997. https://doi. org/10.1177/136216889700100304

LOUW, Kerry J.; DERWING, Tracey M.; ABBOTT, Marilyn L. Teaching pragmatics to L2 learners for the workplace: The job interview. The Canadian Modern Language Review, v. 66, n. 5, p. 739-758, Aug. 2010. https://doi.org/10.3138/cmlr.66.5.739

OLSHTAIN, Olite. Sociocultural competence and language transfer: The case of apology. In KRASHEN, Stephen D.; SCARCELLA, Robin (ed.). Series on Issues on Second Language Research. Rowley: Newbury House, 1983. p. 232-249.

PADILLA CRUZ, Manuel. Metapsychological awareness of comprehension and epistemic vigilance of L2 communication in interlanguage pragmatic development. Journal of Pragmatics, v. 59, n. A, p. 117-135, Dec. 2013. https://doi.org/10.1016/j.pragma.2013.09.005

PERNA, Cristina Lopes. A competência pragmática na realização de pedidos de desculpas em inglês como L2. In: IBANOS, A.; SILVEIRA, J. (ed.). Na Interface Semântica/Pragmática. Porto Alegre: Edipucrs, 2002.

ROMERO TRILLO, Jesús. The pragmatic fossilization of discourse markers in non-native speakers of English. Journal of Pragmatics, v. 34, n. 6, p. 769-784, Jun. 2002. https://doi.org/10.1016/ s0378-2166(02)00022-x

SAFONT JORDÁ, María Pilar. Third Language Learners. Pragmatic Production and Awareness. Clevedon: Multilingual Matters, 2005.

SHIVELY, Rachel L. Developing interactional competence during study abroad: Listener responses in L2 Spanish. System, v. 48, p. 86-98, Feb. 2015. https://doi.org/10.1016/j.system.2014.09.007

TAGUCHI, Naoko. Corpus-informed assessment of comprehension of conversational implicatures in L2 English. TESOL Quarterly, v. 43, n 4, p. 738-749, Dec. 2009. https://doi. org/10.1002/j.1545-7249.2009.tbo0202.x

TAGUCHI, Naoko. Comprehension of conversational implicature. What response times tell us In: TAGUCHI, Naoko; SYKES, Julie M. (ed.). Technology in Interlanguage Pragmatics Research and Teaching. Amsterdam: John Benjamins, 2013. p. 19-41.https://doi.org/10.1075/lllt.36.03tag 
TAGUCHI, Naoko. Instructed pragmatics at a glance: Where instructional studies were, are, and should be going. Language Teaching, v. 48, n 1, p. 1-50, Jan. 2015. https://doi.org/10.1017/ $\underline{\text { s0261444814000263 }}$

TAKAHASHI, Satomi. Noticing in task performance and learning outcomes: A qualitative analysis of instructional effects in interlanguage pragmatics. System, v. 33, n. 3, p. 437-431, Sept. 2005. https://doi.org/10.1016/j.system.2005.06.006

THOMAS, Jenny. Cross-cultural pragmatic failure. Applied Linguistics, v. 4, n 2, p. 91-112, 1983.

WOODFIELD, Helen. Pragmatic variation in learner perception. The role of retrospective verbal report in L2 speech act research. In: FÉLIX-BRASDEFER, Julio C.; KOIKE, Dale A. (ed.). Pragmatic Variation in First and Second Language Contexts: Methodological Issues. Amsterdam: John Benjamins, 2012. p. 209-237. https://doi.org/10.1075/impact.31.08woo 